\title{
Food sources of nutrients among 13-year-old Portuguese adolescents
}

\author{
Joana Araújo ${ }^{1,2, *}$, Milton Severo ${ }^{1,2}$, Carla Lopes ${ }^{1,2}$ and Elisabete Ramos ${ }^{1,2}$ \\ 'Department of Clinical Epidemiology, Preventive Medicine and Public Health and Cardiovascular Research \& \\ Development Unity, University of Porto Medical School, Alameda Prof. Hernâni Monteiro, 4200-3 19 Porto, \\ Portugal: ${ }^{2}$ Institute of Public Health, University of Porto, Porto, Portugal
}

Submitted 3 August 2010: Accepted 28 April 2011: First published online 23 June 2011

\begin{abstract}
Objective: To identify food sources of nutrients in adolescents' diets and to identify differences in food sources according to individual characteristics.

Design: A cross-sectional evaluation was carried out in the 2003/2004 school year. Self-administered questionnaires were used and a physical examination was performed. Diet was evaluated using an FFQ.

Setting: Public and private schools in Porto, Portugal.

Subjects: Adolescents aged 13 years ( $n$ 1522) enrolled at school.

Results: The main sources of energy were starchy foods (26.5\%), dairy (12.5\%) and meat $(12 \cdot 0 \%)$. The major contributors to carbohydrate intake were starchy foods $(38 \cdot 2 \%)$ and fruit $(13 \cdot 8 \%)$ and to protein intake were meat $(28 \cdot 0 \%)$, dairy products $(20 \cdot 3 \%)$, starchy foods $(15 \cdot 3 \%)$ and seafood $(13 \cdot 6 \%)$. The main sources of total fat were meat $(22 \cdot 0 \%)$, starchy foods $(13 \cdot 4 \%)$ and dairy products $(12 \cdot 7 \%)$. Sweets and pastries presented important contributions to energy (11.1\%), carbohydrate $(12 \cdot 4 \%)$, total fat $(13 \cdot 3 \%)$ and saturated fat $(16 \cdot 6 \%)$ intakes. Parental education was inversely associated with the contribution of sweets and pastries to energy, carbohydrate and fat intakes and it was positively associated with the seafood contribution to protein intake.

Conclusions: The major sources of carbohydrates were starchy foods, which also accounted for a quarter of energy intake. Dairy products plus meat accounted for another quarter of energy. Meat was a major source of protein and fats. Sweets and pastries contributed more than $10 \%$ to energy, carbohydrates, total and saturated fat. Parental education was the strongest determinant of food sources and was positively associated with a healthier contribution of food groups.
\end{abstract}

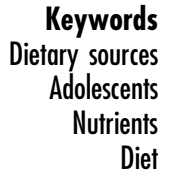

The burden of chronic diseases has been increasing in recent decades. In 2005 these diseases caused an estimated 35 million deaths, which represents $60 \%$ of all deaths worldwide ${ }^{(1)}$. The preventable nature of chronic diseases increases the importance of intervention as an instrument to decrease their burden ${ }^{(2)}$.

Food intake is an important modifiable determinant of many of these diseases and its role throughout the life course in the promotion and maintenance of good health is well established ${ }^{(3)}$. Early interventions are important due to the tracking of behaviours throughout life, which makes it more difficult for people to change their habits in later life ${ }^{(2)}$. Adolescence is a particularly important period because it is a time of greater autonomy, where personal choices and preferences gain priority over family eating habits and in which adolescents progressively gain more control over what, when and where they eat ${ }^{(4,5)}$.

Although a good deal of research has been done into nutrient intake and its relationship to health, from a public health point of view a transition is needed from a nutrient orientation to food-based strategies, preferably derived from existing patterns of food intake in the target population rather than from a theoretical basis ${ }^{(6)}$. Additionally, identification of the main dietary sources of target nutrients is also important, because it allows the identification of differences in the relative contributions of various food groups to nutrient intake ${ }^{(7)}$.

For public health interventions we need to be aware that food intake is determined by the interplay of many factors, such as public policies, food availability, food choices and personal characteristics ${ }^{(8)}$. Socio-economic status is among the most widely described individual characteristic related to food intake, and many studies have shown that people with high education tend to have a diet closer to national or international recommendations ${ }^{(9-11)}$. What is more, age, gender and sport activities are often described as food intake determinants ${ }^{(12)}$. Therefore, the knowledge of differences in diet and specifically in food sources according 
to individual characteristics is important in order to adapt the messages to the general population and thus to provide efficient food-based programmes.

The aim of the present study was to identify the food sources of energy and ten nutrients in the diets of 13-year-old Portuguese adolescents. We also aimed to identify differences in food sources according to individual characteristics.

\section{Methods}

Eligible participants were adolescents born in 1990 and enrolled at public and private schools in Porto, Portugal, during the 2003/2004 school year, as described elsewhere $^{(13)}$. Two thousand seven hundred and eightyseven eligible participants were identified: $2126(76 \cdot 3 \%)$ from public and $661(23 \cdot 7 \%)$ from private schools. Among them, forty-four (1.6\%) could not be reached, 583 $(20.9 \%)$ were refusals (no signed informed consent form was returned) and 2160 (1651 public and 509 private school students) agreed to participate and provided information at least for part of the planned assessment, resulting in an overall participation rate of $77 \cdot 5 \%$, similar in public $(77 \cdot 7 \%)$ and private $(77 \cdot 0 \%)$ schools.

Of the 2160 participants, $247 \mathrm{did}$ not return the home questionnaire and 298 did not fill in the FFQ or were excluded because no information was provided on more than $10 \%$ of food items. A further ninety-three participants were not considered for the current analysis because their total energy intake was more than 3 times the interquartile range or their intake of fruit or vegetables was more than 1.5 times the interquartile range. Thus, the analysis was based on the information of 1522 participants.

The evaluation included two self-administered questionnaires (one completed at home, another at school), comprising information on social, demographic and behavioural characteristics and individual and family history of disease. A physical examination was also performed at school, by a team of experienced nurses, nutritionists and physicians.

As part of the home questionnaire, food intake was recorded using an FFQ regarding the previous 12 months, completed by the adolescents at home with the help of their parents or legal guardians. The FFQ was designed according to Willett and colleagues ${ }^{(14)}$ and adapted for the Portuguese population based on the food items most commonly consumed and with a relevant contribution to the intakes of total energy, protein, fat, carbohydrates, cholesterol, dietary fibre, vitamin A, carotenoids, vitamins $\mathrm{C}$ and $\mathrm{E}, \mathrm{Ca}$, alcohol and caffeine, according to dietary data available for our country, namely the Portuguese food balance sheets and other specific studies ${ }^{(15,16)}$. Foods with similar nutrient composition were grouped together as a single food item. The questionnaire was validated for the adult population by comparison with four $7 \mathrm{~d}$ food records (each one in a different season of the year) ${ }^{(17,18)}$. The FFQ was then adapted for adolescents by including foods more frequently eaten by this age group $^{(19)}$; the adolescents' version comprised ninety-one food items or beverage categories and a frequency section with nine possible responses ranging from never to six or more times daily. It also included an open-ended section for foods not listed in the questionnaire, but eaten at least once weekly.

Food intake data were obtained by multiplying the frequency of consumption of each food item by the nutrient content of the specified portion size. Seasonal variation of food consumption was also considered according to participants' replies. To estimate nutrient intake from the evaluated food intake, we used the software Food Processor Plus version 7 (ESHA Research, Salem, OR, USA) based on values from the US Department of Agriculture. Values for typical Portuguese foods were added, based on the Portuguese tables of food composition, typical recipes and data from previous studies ${ }^{(18)}$. The nutrient content of food items which are usually eaten cooked was estimated by considering cooking and processing.

We defined fourteen food groups: (i) dairy (milk, yoghurt, cheese); (ii) meat (red and white meat, ham, sausages, pepperoni, smoked ham, bacon); (iii) seafood (fresh and canned fish, codfish, molluscs and crustaceans); (iv) eggs; (v) fats and oils (olive and other vegetable oils, margarine and butter); (vi) starchy foods (white bread or rusks and similar toasted products, brown bread, rye bread, cereal flakes, simple biscuits, wholegrain biscuits, rice, pasta, chips and boiled, baked and mashed potatoes); (vii) legumes (beans, chickpeas, peas, etc.); (viii) vegetables (cabbage, spinach, broccoli, onion, carrot, lettuce, peppers, tomato, cucumbers, etc.); (ix) vegetable soup; (x) fruit (fresh fruit, including tropical fruit and fresh fruit juice); (xi) sweets and pastry (other biscuits apart from simple ones, croissants, pastry, doughnuts, cakes, chocolates, chocolate snacks, dairy desserts, ice creams, quince jam, compote, jelly, honey, sugar, candy); (xii) non-alcoholic beverages (soda, juice, fruit juice); (xiii) fast food (pizza, hamburger, mayonnaise, salted snacks); and (xiv) others (canned fruit, nuts, olives, coffee, barley coffee, black tea, green tea, barley).

Food sources were computed for energy, protein, fats (total, saturated, polyunsaturated, monounsaturated and cholesterol), carbohydrates, fibre, $\mathrm{Ca}$ and $\mathrm{Na}$. The amount of a specific nutrient provided by each food was calculated for each person and then each amount was divided by the total intake of that nutrient, resulting in the percentage contribution. The proportion of contribution of each food within the same group was added, to create the contribution of food groups. The results of the contribution of each food group to nutrient intake were presented as medians of the calculated contribution.

Parental education level was measured as the number of successfully completed years of formal schooling; 
in the analysis we used the information from the parent with the highest education level. Each school was considered public if it was state administered or private when it was not administered by local or national government. Parental education level and the type of school were used as indicators of socio-economic class.

We considered as practice of sports any planned, regular exercise, regardless of intensity, that was not part of obligatory curricular activities.

Weight and height were measured following standardized procedures ${ }^{(20)}$. Adolescents were classified according to the age- and sex-specific BMI reference percentiles developed by the US Centers for Disease Control and Prevention ${ }^{(21)}$, considering those at or above the 95th percentile as obese and those at or above the 85 th percentile but below the 95th percentile as overweight.

\section{Statistical analysis}

Statistical analyses were performed using the SPSS statistical software package version $17 \cdot 0$ (SPPS Inc., Chicago, IL, USA).

The $\chi^{2}$ test was used to compare qualitative variables according to gender. The contribution of each food group was presented as median (25th percentile-75th percentile). For each nutrient, food sources according to gender, type of school, parental education, practice of sports and BMI categories were computed only for food groups that provided $10 \%$ or more of the nutrient intake. To estimate the magnitude of the association between the contribution of each food group and participants' characteristics, odds ratios and $95 \%$ confidence intervals were computed using unconditional logistic regression. In order to do this, the contribution of each food group was divided into two categories: one corresponding to those with an intake lower than or equal to the median contribution and the other for those with an intake higher than the median contribution. Final models were adjusted for total energy intake in kilojoules (except in the model of energy sources) as a continuous variable and for gender, type of school, parental education, practice of sports and BMI categories as categorical variables. Logistic regression models included only 1398 individuals, because 124 adolescents had missing information in at least one covariate.

\section{Etbical considerations}

The study was approved by the Ethics Committee of Hospital S. João and written consent was obtained from both legal guardians and adolescents.

\section{Results}

From the 2160 eligible adolescents, we excluded 638 and considered 1522 subjects in the present analysis. The participants excluded were significantly more often male $(52 \cdot 8 \% v \cdot 46 \cdot 5 \%, P=0.008)$, with less educated parents (parental education $>12$ years: $18 \cdot 8 \%$ v. $27 \cdot 7 \%$ ) and mostly from public schools $(84 \cdot 3 \% v \cdot 73 \cdot 1 \%, P<0 \cdot 001)$. In terms of BMI categories and practice of sports, adolescents excluded were not significantly different from those included in the analysis.

The participants' characteristics are described in Table 1. The majority of adolescents attended public schools $(73 \cdot 1 \%)$ and $23 \cdot 4 \%$ had parents with 6 years or less of education, while $27 \cdot 7 \%$ had parents with more than 12 years of education, with no significant differences according to gender. A higher proportion of boys referred to practising sports compared with girls $(62 \cdot 4 \% v .41 \cdot 3 \%$, $P<0 \cdot 001)$. Prevalence of BMI categories did not differ

Table 1 Description and comparison of participants' characteristics, according to gender: 13-year-old adolescents, Porto, Portugal, 2003/2004 school year

\begin{tabular}{|c|c|c|c|c|c|c|c|}
\hline & \multicolumn{2}{|c|}{ Total $(n$ 1522) } & \multicolumn{2}{|c|}{ Girls $(n 815,53 \cdot 5 \%)$} & \multicolumn{2}{|c|}{ Boys ( $n 707,46 \cdot 5 \%)$} & \multirow[b]{2}{*}{$P$} \\
\hline & $n$ & $\%$ & $n$ & $\%$ & $n$ & $\%$ & \\
\hline \multicolumn{8}{|l|}{ School } \\
\hline Public & 1113 & $73 \cdot 1$ & 583 & $71 \cdot 5$ & 530 & $75 \cdot 0$ & \multirow[t]{2}{*}{$0 \cdot 148$} \\
\hline Private & 409 & $26 \cdot 9$ & 232 & $28 \cdot 5$ & 177 & $25 \cdot 0$ & \\
\hline \multicolumn{8}{|c|}{ Parental education (years) } \\
\hline $0-6$ & 345 & $23 \cdot 4$ & 198 & $25 \cdot 0$ & 147 & $21 \cdot 5$ & \multirow[t]{5}{*}{0.077} \\
\hline $7-9$ & 294 & $19 \cdot 9$ & 169 & $21 \cdot 3$ & 125 & $18 \cdot 3$ & \\
\hline $10-12$ & 429 & $29 \cdot 0$ & 213 & $26 \cdot 9$ & 216 & $31 \cdot 6$ & \\
\hline$>12$ & 409 & $27 \cdot 7$ & 213 & $26 \cdot 8$ & 196 & $28 \cdot 6$ & \\
\hline Missing & 45 & & & & & & \\
\hline \multicolumn{8}{|l|}{ Sports } \\
\hline No & 734 & $48 \cdot 9$ & 471 & $58 \cdot 7$ & 263 & $37 \cdot 6$ & \multirow[t]{3}{*}{$<0.001$} \\
\hline Yes & 767 & $51 \cdot 1$ & 331 & $41 \cdot 3$ & 436 & $62 \cdot 4$ & \\
\hline Missing & 21 & & & & & & \\
\hline \multicolumn{8}{|l|}{ BMI percentilet } \\
\hline$<85$ th & 1062 & $72 \cdot 8$ & 585 & $74 \cdot 6$ & 477 & $70 \cdot 7$ & \multirow[t]{4}{*}{$0 \cdot 223$} \\
\hline$\geq 85$ th and $<95$ th & 243 & $16 \cdot 6$ & 120 & $15 \cdot 3$ & 123 & $18 \cdot 2$ & \\
\hline$\geq 95$ th & 154 & $10 \cdot 6$ & 79 & $10 \cdot 1$ & 75 & $11 \cdot 1$ & \\
\hline Missing & 63 & & & & & & \\
\hline
\end{tabular}

tAccording to Centers for Disease Control and Prevention ${ }^{(21)}$. 
according to gender: $15 \cdot 3 \%$ of girls and $18 \cdot 2 \%$ of boys were overweight and $10 \cdot 1 \%$ of girls and $11 \cdot 1 \%$ of boys were obese.

The major contributors to energy intake were starchy foods $(26.5 \%)$, followed by dairy foods $(12.5 \%)$ and meat (12.0\%; Table 2). Sweets and pastry accounted for about $10 \%$ of energy intake. The three most important sources of carbohydrates were starchy foods (38.2\%), fruit $(13 \cdot 8 \%)$ and sweets and pastry $(12 \cdot 4 \%)$. Meat was the most important source of protein intake and its contribution was more than double that of seafood $(28.0 \%$ v. $13.6 \%$, respectively). The second protein source was the dairy group (20.3\%). The major contributor to total fat intake was meat $(22 \cdot 0 \%)$, with starchy foods, dairy and sweets and pastry each accounting for about $13 \%$ of the total fat intake. Meat was one of the highest sources of all types of fat, overtaken in saturated fat $(23.0 \%)$ only by dairy foods and by starchy foods in polyunsaturated fat $(27 \cdot 1 \%)$. The two major contributors to fibre were starchy foods and fruit (38.0 \% and $22 \cdot 1 \%$, respectively). The leading source of $\mathrm{Ca}$ intake was dairy foods, providing almost $60 \%$ of the total intake. Finally, the most important sources of $\mathrm{Na}$ intake were starchy foods, accounting for $34 \cdot 6 \%$, followed by meat $(14 \cdot 9 \%)$ and dairy foods $(14 \cdot 1 \%)$.

Comparing food contributions according to gender, we found statistically significant differences in the contribution of starchy foods to energy (respectively among girls and boys: $25 \cdot 8 \% v \cdot 27 \cdot 5 \%, P=0 \cdot 002$ ), carbohydrate $(36 \cdot 8 \% v \cdot 39 \cdot 3 \%, P=0.001)$ and $\mathrm{Na}(34 \cdot 0 \% v .35 \cdot 9 \%$, $P=0.006)$ intakes; and the contribution of fruit to carbohydrates $(14 \cdot 6 \% v \cdot 13 \cdot 2 \%, P<0 \cdot 001)$.

For all nutrients, Tables 3 and 4 show the association between food sources that provided $10 \%$ or more of the total intake of each nutrient according to some participant characteristics, presented as odds ratio adjusted for gender, type of school, parental education, sports, BMI and total energy intake. In comparison to girls, boys were more likely to have a higher contribution from starchy foods, significant for energy, carbohydrates and fibre; and to have a higher contribution from meat to saturated fat and monounsaturated fat intakes. However, being a boy was inversely associated with the contribution of fruit to carbohydrate intake (Table 3).

Considering the indicators of socio-economic class, adolescents from private schools presented a significantly lower contribution from sweets and pastry to carbohydrate intake and a lower contribution from starchy foods to fibre intake. Parental education was inversely associated with the contribution of sweets and pastry, significant for energy, carbohydrates and total, saturated and monounsaturated fats. It was also inversely associated with the contribution of starchy foods to total and monounsaturated fat intakes and with meat contribution to $\mathrm{Na}$ intake. A positive association was found between parental education and: seafood contribution, significant for protein and cholesterol; dairy contribution to $\mathrm{Na}$ and
艺

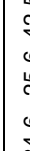

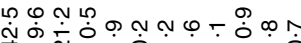

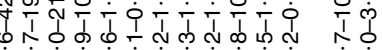
(a) $0 \div 0 m 0 \div 0000+4$

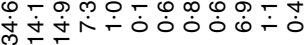
ํำ

ํํำ ஸி ஸि ம்

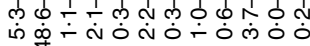

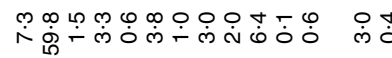

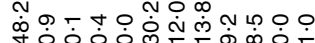

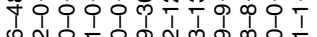

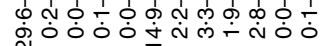
OronormonoOm

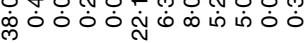

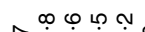

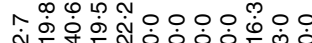

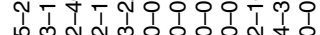

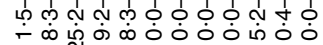
0nturooson ஸे ๑) 0 ம்

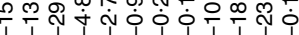

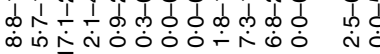
언

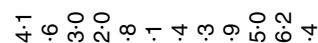

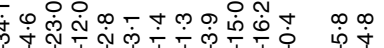

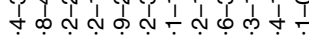

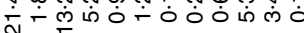
- OnNLONNOmON ลें त

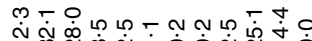

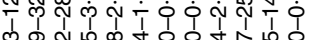

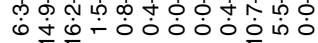
$000 m m \hat{\varphi}-\bar{m} 0 m 0$

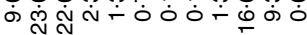
an

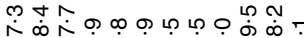
Tテ ฟे

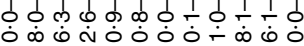
Anootmm Mm mo

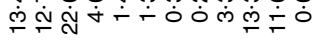

Hot フ

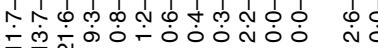

м м

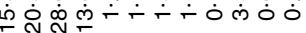
- लn

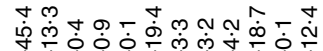
1.

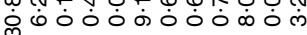

n+m

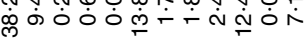

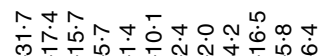

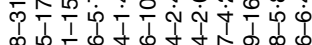

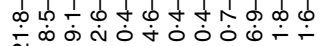

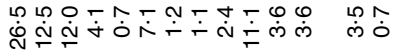
$\hat{+}$
$\dot{+}$
$\stackrel{9}{9}$
$\dot{1}$ लํํ เீ $\dot{\hat{\imath}} \dot{\Lambda}$ ํํำ $\stackrel{\varphi}{\circ} \stackrel{0}{-}$ 웅 $\begin{array}{ll}0 \\ 1 & 0 \\ 1 & 1 \\ \text { in } & 0\end{array}$ 官 พ ம் $\begin{array}{cc}1 & 1 \\ & 0 \\ 0 & 0\end{array}$ ஸे ஸें $\stackrel{\leftrightarrow}{\dot{0}}$ ழ் ஸ่ 숭 암 $\varphi$
1
0
0 ஸீர 0 o $\stackrel{1}{0}$ ம் ஸे 人 No ப욤

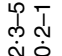

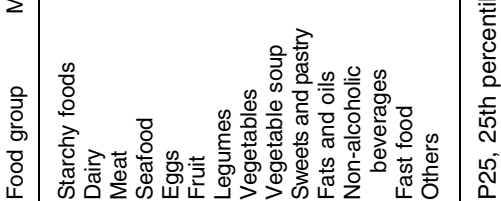


Table 3 Adjusted odds ratio of having a highert $v$. a lower contribution of the indicated food groups to daily energy and nutrient intakes: 13-year-old adolescents ( $n$ 1398), Porto, Portugal, 2003/ 2004 school year

\begin{tabular}{|c|c|c|c|c|c|c|c|c|c|c|}
\hline & \multirow{2}{*}{\multicolumn{2}{|c|}{$\frac{\text { Gender } \ddagger}{\text { Boys }}$}} & \multirow{2}{*}{\multicolumn{2}{|c|}{$\begin{array}{l}\text { School§ } \\
\text { Private }\end{array}$}} & \multicolumn{6}{|c|}{ Parental education (years)\| } \\
\hline & & & & & \multicolumn{2}{|c|}{$7-9$} & \multicolumn{2}{|c|}{$10-12$} & \multicolumn{2}{|c|}{$>12$} \\
\hline & Adjusted ORণ & $95 \% \mathrm{Cl}$ & Adjusted OR $\uparrow$ & $95 \% \mathrm{Cl}$ & Adjusted OR & $95 \% \mathrm{Cl}$ & Adjusted OR $\uparrow$ & $95 \% \mathrm{Cl}$ & Adjusted OR $\uparrow$ & $95 \% \mathrm{Cl}$ \\
\hline \multicolumn{11}{|l|}{ Starchy foods } \\
\hline Energy & $1 \cdot 26^{*}$ & $1 \cdot 02,1 \cdot 57$ & 0.99 & $0 \cdot 77,1 \cdot 28$ & $1 \cdot 22$ & $0.88,1 \cdot 68$ & $1 \cdot 11$ & $0 \cdot 82,1 \cdot 51$ & 1.05 & $0.77,1.45$ \\
\hline Carbohydrates & $1.34^{*}$ & $1 \cdot 07,1.66$ & 0.93 & $0 \cdot 72,1 \cdot 19$ & 1.00 & $0 \cdot 72,1 \cdot 38$ & $1 \cdot 31$ & $0 \cdot 97,1 \cdot 78$ & $1 \cdot 44^{\star}$ & $1.04,1.98$ \\
\hline Protein & $1 \cdot 22$ & $0.98,1.52$ & 0.99 & $0 \cdot 76,1 \cdot 26$ & $1 \cdot 04$ & $0 \cdot 75,1 \cdot 44$ & 0.94 & $0 \cdot 69,1 \cdot 27$ & 0.95 & $0.69,1.31$ \\
\hline Fibre & $1 \cdot 28^{\star}$ & $1 \cdot 03,1 \cdot 58$ & $0 \cdot 77^{\star}$ & $0.60,0.99$ & 0.96 & $0 \cdot 70,1 \cdot 33$ & $1 \cdot 12$ & $0.83,1 \cdot 52$ & $0 \cdot 75$ & $0.55,1.02$ \\
\hline Total fat & 1.06 & $0 \cdot 85,1 \cdot 32$ & 1.02 & $0 \cdot 80,1 \cdot 32$ & $1 \cdot 25$ & $0.90,1 \cdot 74$ & 0.78 & $0.58,1.06$ & $0.53^{\star}$ & $0.38,0.73$ \\
\hline MUFA & 0.97 & $0 \cdot 85,1 \cdot 32$ & 1.05 & $0 \cdot 82,1 \cdot 34$ & $1 \cdot 26$ & $0.91,1 \cdot 75$ & $0.73^{\star}$ & $0.54,0.99$ & $0.52^{*}$ & $0.38,0.71$ \\
\hline PUFA & 1.08 & $0.87,1.34$ & 0.97 & $0 \cdot 76,1 \cdot 24$ & $1 \cdot 37$ & $0.99,1.90$ & 0.84 & $0 \cdot 62,1 \cdot 14$ & $0 \cdot 76$ & $0.55,1.03$ \\
\hline $\mathrm{Na}$ & $1 \cdot 21$ & $0.97,1.50$ & 1.08 & $0 \cdot 84,1 \cdot 38$ & $1 \cdot 30$ & $0.94,1 \cdot 80$ & $1 \cdot 22$ & $0 \cdot 90,1 \cdot 65$ & $1 \cdot 57^{\star}$ & $1 \cdot 14,2 \cdot 17$ \\
\hline \multicolumn{11}{|l|}{ Dairy } \\
\hline Energy & 0.94 & $0 \cdot 76,1 \cdot 16$ & 1.09 & $0 \cdot 85,1 \cdot 40$ & $0 \cdot 78$ & $0.57,1.08$ & 1.06 & $0 \cdot 78,1 \cdot 43$ & $1 \cdot 19$ & $0.86,1.63$ \\
\hline Protein & 0.89 & $0 \cdot 72,1 \cdot 11$ & $1 \cdot 12$ & $0 \cdot 88,1.44$ & 0.88 & $0 \cdot 63,1 \cdot 21$ & 1.05 & $0 \cdot 78,1 \cdot 42$ & 1.06 & $0.77,1.45$ \\
\hline Total fat & 1.02 & $0.82,1 \cdot 26$ & $1 \cdot 11$ & $0 \cdot 87,1.43$ & 0.96 & $0 \cdot 70,1 \cdot 32$ & $1 \cdot 21$ & $0 \cdot 89,1 \cdot 64$ & $1 \cdot 33$ & $0.97,1.84$ \\
\hline Cholesterol & $1 \cdot 13$ & $0.91,1.40$ & $1 \cdot 17$ & $0.91,1.50$ & 1.01 & $0.73,1 \cdot 40$ & $1 \cdot 32$ & $0.97,1 \cdot 79$ & $1 \cdot 49^{\star}$ & $1 \cdot 09,2 \cdot 04$ \\
\hline SFA & 1.04 & $0.84,1 \cdot 30$ & $1 \cdot 20$ & $0.94,1.54$ & 0.83 & $0 \cdot 60,1 \cdot 14$ & $1 \cdot 15$ & $0.85,1.56$ & 1.20 & $0.88,1.64$ \\
\hline $\mathrm{Ca}$ & $1 \cdot 12$ & $0.90,1.39$ & 1.05 & $0 \cdot 82,1 \cdot 34$ & 0.91 & $0 \cdot 66,1 \cdot 26$ & $1 \cdot 12$ & $0 \cdot 83,1 \cdot 51$ & $1 \cdot 22$ & $0.90,1.67$ \\
\hline $\mathrm{Na}$ & 0.93 & $0 \cdot 75,1 \cdot 16$ & $1 \cdot 17$ & $0.91,1.50$ & $1 \cdot 00$ & $0 \cdot 72,1 \cdot 38$ & $1 \cdot 25$ & $0.92,1.69$ & $1 \cdot 61^{*}$ & $1 \cdot 17,2 \cdot 20$ \\
\hline \multicolumn{11}{|l|}{ Meat } \\
\hline Energy & $1 \cdot 22$ & $0.98,1.51$ & 0.97 & $0 \cdot 75,1 \cdot 24$ & 0.99 & $0 \cdot 72,1 \cdot 37$ & 0.82 & $0 \cdot 61,1 \cdot 12$ & 0.99 & $0.72,1.36$ \\
\hline Protein & $1 \cdot 15$ & $0.93,1.44$ & 1.04 & $0 \cdot 81,1 \cdot 34$ & 1.08 & $0.78,1.50$ & $0.69^{*}$ & $0.51,0.94$ & 0.83 & $0 \cdot 60,1 \cdot 15$ \\
\hline Total fat & $1 \cdot 26^{*}$ & $1.01,1.57$ & 0.99 & $0 \cdot 77,1 \cdot 28$ & 1.00 & $0.72,1.39$ & 0.77 & $0.57,1.05$ & 1.00 & $0.72,1.38$ \\
\hline Cholesterol & 1.04 & $0.84,1.29$ & 1.08 & $0.85,1.39$ & 0.98 & $0 \cdot 71,1 \cdot 36$ & $0 \cdot 65^{\star}$ & $0 \cdot 48,0.88$ & $0 \cdot 80$ & $0.58,1.09$ \\
\hline SFA & $1 \cdot 29^{\star}$ & $1 \cdot 04,1 \cdot 61$ & 0.88 & $0 \cdot 69,1 \cdot 13$ & 1.07 & $0.77,1.48$ & 0.92 & $0 \cdot 68,1 \cdot 26$ & $1 \cdot 32$ & $0.97,1.82$ \\
\hline MUFA & $1 \cdot 24^{\star}$ & $1 \cdot 00,1.54$ & 0.97 & $0 \cdot 76,1 \cdot 24$ & 0.98 & $0 \cdot 71,1 \cdot 36$ & $0.72^{*}$ & $0.53,0.97$ & $0 \cdot 86$ & $0 \cdot 63,1 \cdot 17$ \\
\hline PUFA & $1 \cdot 14$ & $0.92,1.42$ & 1.05 & $0 \cdot 82,1 \cdot 35$ & $1 \cdot 00$ & $0 \cdot 72,1 \cdot 39$ & $0 \cdot 80$ & $0.59,1.09$ & 0.99 & $0.72,1.35$ \\
\hline $\mathrm{Na}$ & $1 \cdot 14$ & $0.91,1.42$ & 0.96 & $0 \cdot 75,1 \cdot 24$ & $0.66^{*}$ & $0.48,0.92$ & $0.56^{*}$ & $0 \cdot 41,0 \cdot 76$ & $0.48^{\star}$ & $0.35,0.66$ \\
\hline \multicolumn{11}{|l|}{ Seafood } \\
\hline Protein & $1 \cdot 02$ & $0 \cdot 82,1 \cdot 27$ & $1 \cdot 02$ & $0 \cdot 79,1 \cdot 30$ & $1 \cdot 31$ & $0.94,1 \cdot 81$ & $1 \cdot 43^{\star}$ & $1 \cdot 06,1 \cdot 94$ & $1.38^{*}$ & $1.00,1.90$ \\
\hline Cholesterol & 0.91 & $0 \cdot 73,1 \cdot 13$ & $1 \cdot 11$ & $0.87,1.42$ & $1 \cdot 51^{*}$ & $1 \cdot 08,2 \cdot 09$ & $1 \cdot 83^{*}$ & $1 \cdot 34,2 \cdot 49$ & $1.98^{*}$ & $1 \cdot 45,2 \cdot 72$ \\
\hline \multicolumn{11}{|l|}{ Eggs } \\
\hline \multirow{2}{*}{\multicolumn{11}{|c|}{ Fruit }} \\
\hline & & & & & & & & & & \\
\hline Carbohydrates & $0 \cdot 76^{\star}$ & $0.61,0.95$ & $1 \cdot 12$ & $0 \cdot 87,1 \cdot 44$ & 1.02 & $0 \cdot 73,1 \cdot 41$ & 1.02 & $0 \cdot 75,1 \cdot 38$ & $1 \cdot 99^{*}$ & $1 \cdot 44,2 \cdot 75$ \\
\hline Fibre & $0 \cdot 82$ & $0 \cdot 66,1 \cdot 02$ & 1.06 & $0 \cdot 83,1 \cdot 36$ & 1.05 & $0 \cdot 76,1 \cdot 46$ & 0.96 & $0 \cdot 71,1 \cdot 29$ & $1 \cdot 48^{*}$ & $1 \cdot 08,2 \cdot 02$ \\
\hline \multicolumn{11}{|l|}{ Sweets and pastry } \\
\hline Energy & 1.01 & $0 \cdot 81,1 \cdot 26$ & 0.81 & $0 \cdot 62,1 \cdot 04$ & 0.75 & $0.54,1.04$ & $0.58^{\star}$ & $0.42,0.79$ & $0 \cdot 48^{*}$ & $0.35,0.67$ \\
\hline Carbohydrates & 0.98 & $0.79,1 \cdot 23$ & $0.77^{\star}$ & $0.60,0.99$ & $0 \cdot 78$ & $0 \cdot 56,1 \cdot 10$ & $0.56^{*}$ & $0 \cdot 41,0.77$ & $0.46^{\star}$ & $0.33,0.64$ \\
\hline Total fat & 1.04 & $0 \cdot 83,1 \cdot 29$ & 0.91 & $0 \cdot 70,1 \cdot 17$ & $0.72^{\star}$ & $0.51,1.00$ & $0.54^{*}$ & $0.40,0.74$ & $0.52^{\star}$ & $0.37,0.72$ \\
\hline SFA & 0.99 & $0 \cdot 80,1 \cdot 23$ & 0.98 & $0 \cdot 76,1 \cdot 25$ & 0.79 & $0 \cdot 57,1 \cdot 10$ & $0.63^{*}$ & $0 \cdot 46,0 \cdot 86$ & $0.54^{\star}$ & $0 \cdot 39,0 \cdot 74$ \\
\hline MUFA & 1.09 & $0 \cdot 87,1 \cdot 35$ & 0.90 & $0 \cdot 70,1 \cdot 15$ & $0 \cdot 63^{\star}$ & $0.45,0.88$ & $0 \cdot 49^{\star}$ & $0.36,0.67$ & $0.46^{*}$ & $0.33,0.63$ \\
\hline \multicolumn{11}{|l|}{ Fats and oils } \\
\hline Total fat & $1 \cdot 16$ & $0.93,1.44$ & 0.90 & $0 \cdot 70,1 \cdot 15$ & $1 \cdot 61^{*}$ & $1 \cdot 16,2 \cdot 24$ & $2 \cdot 00^{*}$ & $1 \cdot 47,2 \cdot 72$ & $2 \cdot 13^{*}$ & $1 \cdot 54,2 \cdot 95$ \\
\hline MUFA & 1.09 & $0.88,1.35$ & 0.85 & $0 \cdot 66,1 \cdot 08$ & $1 \cdot 64^{*}$ & $1 \cdot 18,2 \cdot 27$ & $2 \cdot 25^{\star}$ & $1 \cdot 65,3.06$ & $2 \cdot 18^{\star}$ & $1.59,2.99$ \\
\hline
\end{tabular}

OR and $95 \% \mathrm{Cl}$ are statistically significant.

Have a food contribution above the median

Reference class: girls.

Reference class: public school.

Reference class: $0-6$ years of education

OR adjusted for total energy intake, as a continuous variable, and for gender, type of school, parental education, sports and BMI as categorical variables. 
Table 4 Adjusted odds ratio having a highert $v$. a lower contribution of the indicated food groups to daily energy and nutrient intakes: 13-year-old adolescents ( $n$ 1398), Porto, Portugal, 2003/2004 school year

\begin{tabular}{|c|c|c|c|c|c|c|}
\hline & \multirow{2}{*}{\multicolumn{2}{|c|}{$\frac{\text { Sportsł }}{\text { Yes }}$}} & \multicolumn{4}{|c|}{ BMI percentile§ } \\
\hline & & & \multicolumn{2}{|c|}{$\geq 85$ th and $<95$ th } & \multicolumn{2}{|c|}{$\geq 95$ th } \\
\hline & Adjusted OR\| & $95 \% \mathrm{Cl}$ & Adjusted OR\| & $95 \% \mathrm{Cl}$ & Adjusted OR\| & $95 \% \mathrm{Cl}$ \\
\hline \multicolumn{7}{|l|}{ Starchy foods } \\
\hline Energy & $1 \cdot 12$ & $0 \cdot 90,1 \cdot 40$ & $1 \cdot 11$ & $0 \cdot 83,1 \cdot 48$ & $1 \cdot 18$ & $0.84,1.67$ \\
\hline Carbohydrates & $1 \cdot 04$ & $0 \cdot 83,1 \cdot 30$ & $1 \cdot 04$ & $0 \cdot 78,1 \cdot 40$ & $1 \cdot 06$ & $0.75,1.51$ \\
\hline Protein & $1 \cdot 23$ & $0.98,1.54$ & $1 \cdot 03$ & $0 \cdot 77,1 \cdot 38$ & 0.88 & $0 \cdot 62,1 \cdot 25$ \\
\hline Fibre & $1 \cdot 08$ & $0 \cdot 86,1 \cdot 34$ & 0.94 & $0 \cdot 71,1 \cdot 26$ & 0.84 & $0 \cdot 60,1 \cdot 19$ \\
\hline Total fat & $1 \cdot 00$ & $0 \cdot 80,1 \cdot 25$ & $1 \cdot 02$ & $0 \cdot 76,1 \cdot 36$ & $1 \cdot 22$ & $0.86,1 \cdot 74$ \\
\hline MUFA & 0.99 & $0 \cdot 79,1 \cdot 23$ & $0 \cdot 91$ & $0 \cdot 68,1 \cdot 21$ & $1 \cdot 18$ & $0.83,1.68$ \\
\hline PUFA & $1 \cdot 01$ & $0 \cdot 81,1 \cdot 26$ & 0.79 & $0.59,1.05$ & $1 \cdot 07$ & $0.76,1.52$ \\
\hline $\mathrm{Na}$ & $1 \cdot 30^{*}$ & $1 \cdot 04,1 \cdot 62$ & $0 \cdot 84$ & $0.63,1 \cdot 13$ & $0 \cdot 87$ & $0 \cdot 61,1 \cdot 24$ \\
\hline \multicolumn{7}{|l|}{ Dairy } \\
\hline Energy & $1 \cdot 16$ & $0.93,1.45$ & 0.99 & $0 \cdot 75,1 \cdot 33$ & $1 \cdot 18$ & $0.83,1.67$ \\
\hline Protein & $1 \cdot 04$ & $0 \cdot 83,1 \cdot 30$ & $1 \cdot 12$ & $0 \cdot 84,1 \cdot 49$ & $1 \cdot 05$ & $0.74,1.48$ \\
\hline Total fat & $1 \cdot 20$ & $0.96,1.50$ & $0 \cdot 86$ & $0 \cdot 64,1 \cdot 15$ & $1 \cdot 00$ & $0.71,1.42$ \\
\hline Cholesterol & 1.09 & $0.87,1 \cdot 36$ & 0.92 & $0 \cdot 69,1 \cdot 23$ & 0.90 & $0 \cdot 64,1 \cdot 28$ \\
\hline SFA & $1 \cdot 25^{\star}$ & $1 \cdot 00,1 \cdot 56$ & 0.91 & $0 \cdot 69,1 \cdot 22$ & 0.98 & $0 \cdot 70,1 \cdot 39$ \\
\hline $\mathrm{Ca}$ & $1 \cdot 06$ & $0.85,1.32$ & $1 \cdot 17$ & $0.88,1.56$ & 1.06 & $0.75,1.50$ \\
\hline $\mathrm{Na}$ & $1 \cdot 06$ & $0 \cdot 85,1 \cdot 33$ & $1 \cdot 02$ & $0 \cdot 77,1 \cdot 37$ & $1 \cdot 20$ & $0 \cdot 85,1 \cdot 71$ \\
\hline \multicolumn{7}{|l|}{ Meat } \\
\hline Energy & 0.96 & $0 \cdot 77,1 \cdot 20$ & $1 \cdot 03$ & $0 \cdot 77,1 \cdot 37$ & $1 \cdot 44^{*}$ & $1 \cdot 01,2 \cdot 04$ \\
\hline Protein & $1 \cdot 04$ & $0 \cdot 83,1 \cdot 30$ & 0.87 & $0 \cdot 65,1 \cdot 16$ & $1 \cdot 11$ & $0.78,1.58$ \\
\hline Total fat & 0.95 & $0 \cdot 76,1 \cdot 19$ & $1 \cdot 16$ & $0.86,1 \cdot 55$ & $1 \cdot 33$ & $0.93,1.90$ \\
\hline Cholesterol & $1 \cdot 07$ & $0 \cdot 86,1 \cdot 33$ & $1 \cdot 05$ & $0 \cdot 79,1 \cdot 39$ & $1 \cdot 10$ & $0.78,1.56$ \\
\hline SFA & 0.90 & $0 \cdot 72,1 \cdot 13$ & $1 \cdot 29$ & $0.96,1.72$ & $1 \cdot 27$ & $0.89,1 \cdot 80$ \\
\hline MUFA & 0.89 & $0 \cdot 72,1 \cdot 11$ & 0.95 & $0 \cdot 71,1 \cdot 26$ & $1 \cdot 22$ & $0 \cdot 86,1 \cdot 73$ \\
\hline PUFA & $1 \cdot 07$ & $0 \cdot 86,1 \cdot 34$ & $1 \cdot 01$ & $0 \cdot 76,1 \cdot 35$ & $1 \cdot 10$ & $0.78,1.57$ \\
\hline $\mathrm{Na}$ & $0 \cdot 88$ & $0 \cdot 70,1 \cdot 10$ & $1 \cdot 17$ & $0 \cdot 88,1 \cdot 57$ & $1 \cdot 31$ & $0.92,1 \cdot 87$ \\
\hline \multicolumn{7}{|l|}{ Seafood } \\
\hline Protein & 1.06 & $0 \cdot 84,1 \cdot 32$ & $0 \cdot 84$ & $0 \cdot 63,1 \cdot 13$ & 0.92 & $0 \cdot 65,1 \cdot 30$ \\
\hline Cholesterol & $1 \cdot 14$ & $0.92,1.42$ & 0.92 & $0 \cdot 69,1 \cdot 23$ & $1 \cdot 04$ & $0.73,1.47$ \\
\hline \multicolumn{7}{|l|}{ Eggs } \\
\hline Cholesterol & $0 \cdot 91$ & $0 \cdot 73,1 \cdot 14$ & $1 \cdot 00$ & $0 \cdot 75,1 \cdot 33$ & $1 \cdot 10$ & $0.78,1 \cdot 56$ \\
\hline \multicolumn{7}{|l|}{ Fruit } \\
\hline Carbohydrates & $1 \cdot 25^{\star}$ & $1 \cdot 00,1 \cdot 56$ & $1 \cdot 39^{\star}$ & $1 \cdot 04,1 \cdot 87$ & $1 \cdot 34$ & $0.94,1.90$ \\
\hline Fibre & $1 \cdot 07$ & $0 \cdot 86,1 \cdot 34$ & $1 \cdot 24$ & $0.94,1 \cdot 66$ & $1 \cdot 43^{*}$ & $1 \cdot 01,2 \cdot 03$ \\
\hline \multicolumn{7}{|l|}{ Sweets and pastry } \\
\hline Energy & $0 \cdot 87$ & $0 \cdot 69,1 \cdot 09$ & $0 \cdot 66^{\star}$ & $0.50,0.89$ & $0 \cdot 41^{*}$ & $0.28,0.59$ \\
\hline Carbohydrates & 0.80 & $0 \cdot 63,1 \cdot 00$ & $0 \cdot 58^{\star}$ & $0 \cdot 43,0.78$ & $0 \cdot 42^{\star}$ & $0.29,0.62$ \\
\hline Total fat & 0.94 & $0 \cdot 75,1 \cdot 18$ & 0.86 & $0 \cdot 64,1 \cdot 15$ & $0 \cdot 48^{\star}$ & $0.34,0 \cdot 70$ \\
\hline SFA & 0.94 & $0 \cdot 75,1 \cdot 17$ & 0.82 & $0 \cdot 62,1 \cdot 10$ & $0 \cdot 62^{*}$ & $0.43,0.88$ \\
\hline MUFA & 0.93 & $0 \cdot 74,1 \cdot 16$ & $0 \cdot 73^{\star}$ & $0.55,0.98$ & $0 \cdot 47^{\star}$ & $0.32,0.68$ \\
\hline \multicolumn{7}{|l|}{ Fats and oils } \\
\hline Total fat & $0.79^{\star}$ & $0.63,0.99$ & 1.06 & $0 \cdot 80,1 \cdot 41$ & 0.91 & $0.64,1 \cdot 30$ \\
\hline MUFA & $0 \cdot 88$ & $0 \cdot 71,1 \cdot 10$ & $1 \cdot 16$ & $0.88,1.55$ & 0.95 & $0.67,1.35$ \\
\hline
\end{tabular}

*OR and $95 \% \mathrm{Cl}$ are statistically significant.

thave a food contribution above the median.

†Reference class: do not practice sports.

$\S$ Reference class: $\mathrm{BMI}<85$ th percentile.

IOR adjusted for total energy intake, as a continuous variable, and for gender, type of school, parental education, sports and BMI as categorical variables.

cholesterol intakes; the contribution of eggs to cholesterol intake; and the contribution of starchy foods to carbohydrate and $\mathrm{Na}$ intakes (Table 3).

Adolescents who referred to practising sports were more likely to have a significantly higher contribution of: starchy foods to $\mathrm{Na}$ intake; fruit to carbohydrate intake; and dairy foods to saturated fat intake (Table 4). On the other hand, the practice of sports was inversely associated with the contribution of fats and oils to total fat intake.

BMI was inversely associated with the contribution of sweets and pastry, significant for energy, carbohydrates and for total, saturated and monounsaturated fats. It was directly associated with the contribution of meat to energy intake and with the contribution of fruit to carbohydrate and fibre intakes (Table 4).

\section{Discussion}

In this sample of 13-year-old adolescents, starchy foods were one of the main food sources of the nutrients analysed, presenting an important contribution to energy, 
carbohydrates, total and polyunsaturated fat, fibre and $\mathrm{Na}$. The high contribution of this group to fat intake or even to Na intake could seem unexpected; however, this is because cooked food is taken into account (for instance potatoes, pasta and rice), which contains added fats and salt. Additionally, processed foods, such as bread, biscuits and breakfast cereals, have a high salt content, since it is used as a preservative. Sweets and pastry, a group of high-energy-dense foods, presented an undesirably high contribution to energy intake, carbohydrates and fats. This contribution indicates the high consumption of these foods, which is in fact evident in data on Portuguese household food availability using the Data Food Networking (DAFNE) classification system ${ }^{(22)}$, which showed an increase in the household availability of bakery and sugar products between 1990 and $2000^{(23)}$.

The contribution of fish to the diet of the adolescents in our sample was much lower than that of meat, since we found that the contribution of seafood to protein intake was less than half that of meat. Additionally, the contribution of seafood to the intake of total and polyunsaturated fats was much lower than the contribution of meat, which could be undesirable because of the known benefits of fats from fish ${ }^{(24)}$ regarding specific fatty acids, which were not analysed separately in this work. Since Portugal presented the highest food availability of fish in 1990 and 1995 among European countries participating in the DAFNE project ${ }^{(25)}$, our data may indicate that in other European countries the contribution of seafood to different nutrients may be much lower.

This high consumption of meat in our sample explains the high contribution of this food group to the intake of MUFA, even in a country with a high consumption of olive oil which is richer in this type of fat, but much less consumed than meat.

The importance of the consumption of fruit and vegetables is widely recognized. Since 1990 the WHO has recommended a minimum consumption of approximately 5 portions of fruit or/and vegetables daily ${ }^{(26)}$, with a higher consumption of vegetables than fruit. However, in adolescents, more fruit was consumed than vegetables, since its contribution to fibre intake $(22 \cdot 1 \%)$ was similar to or even higher than the contribution of vegetables ( $13 \cdot 2 \%$, including the $5 \cdot 2 \%$ provided by vegetable soup).

When we made a comparison with the food sources of Portuguese adults ${ }^{(27)}$ assessed using a similar methodology of nutrient intake evaluation, our results were, in general, similar. Only few differences were found: while in adults seafood was the second highest source of protein, in adolescents it was the fourth; adolescents presented a higher contribution of sweets and pastry to total and saturated fat intakes and a lower contribution of soup and seafood to $\mathrm{Na}$ intake. All these differences were expected, based on the general reported differences according to age, with a lower consumption of seafood and soup and a higher consumption of sweets and pastry in younger ages ${ }^{(28-30)}$.
Analysis of food sources according to individuals' characteristics showed an important effect of socio-economic class. Adolescents of high socio-economic class seemed to present a healthier contribution of food groups to nutrient intake. In general, adolescents whose parents were more educated presented higher contributions from high-nutrientdense foods (fruit and vegetables) to carbohydrate and fibre intakes and lower contributions of high-energy-dense foods (sweets and pastry) to energy, carbohydrates and fats. Our results are in accordance with previous data from a representative sample of Portuguese adults, which showed that a high educational level was related to a higher frequency of milk, vegetable soup, vegetable, fruit and fish consumption $^{(31)}$, and also with data from other countries ${ }^{(10,32)}$.

Overweight and obese adolescents presented a lower contribution of sweets and pastry to energy and other nutrients. This could be related to the previously described effect that obese adolescents under-report their food intake more than their normal-weight counterparts ${ }^{(33)}$. On the other hand, as the present study was cross-sectional, these results might also be due to reverse causation, in which the temporal relationship between diet and weight is distorted - possibly overweight adolescents decreased food intake, and particularly some high-energy-dense foods, in order to lose weight.

In our work we used an FFQ and this method has some limitations in assessing dietary intake, such as using a predetermined food list that might not be representative of foods eaten by a specific population ${ }^{(34)}$. Nevertheless, we believe that this possible bias had a very low effect, because this FFQ was validated for the adult population ${ }^{(17,18)}$ and furthermore some foods or food groups eaten more frequently by the adolescent age group were included in the questionnaire. Moreover, in an open section, adolescents were also encouraged to list foods eaten at least once weekly that were not in the FFQ. However, the nonvalidation of the FFQ in adolescents may have led to some bias in the assessment of dietary intake and consequently to misclassification, and an overestimation was to be expected, particularly for fruit and vegetables, which are perceived as healthy and socially acceptable foods ${ }^{(35)}$.

Another limitation of the FFQ is the reliance on participants' recall and the requirement of motivated participants, especially in self-administered questionnaires ${ }^{(34)}$. Our FFQ was self-administered, but adolescents were given oral instructions on filling it in and written instructions were also sent home along with the questionnaire. Moreover, adolescents completed it with the help of their parents or legal guardians, which may have improved the quality of information. However, the extent to which parents might have an influence on overestimating healthy foods (according to what is socially acceptable) and on underestimating unhealthy foods is unknown.

Although the FFQ was filled in with the help of the parents, portion size information was not collected because Willett ${ }^{(34)}$ suggests that to record information on 
portion size does not substantially improve the assessment of dietary intake, which may be even more relevant in adolescents who may have difficulty in estimating the portions and frequently ignore these questions when questionnaires are self-administered ${ }^{(36)}$.

Apart from the exclusions by total energy intake, we also excluded those participants with an intake of fruit or vegetables equal to or higher than 1.5 times the interquartile range. This decision was taken because of the expected overestimation in their consumption related with this method ${ }^{(37)}$. Moreover, fruit and vegetables intake is assessed by a long list of different items, some of them consumed only seasonally, making the evaluation of their intake more difficult and more likely to be overestimated. Additionally, as they are not energy-dense foods, overestimating them might not be reflected in a high total energy intake so those participants would not be excluded by the criterion of the total energy intake.

Adolescents included in the present analysis were significantly more often female, with highly educated parents and more from private schools, compared with those excluded. This over-representation of adolescents from high social classes may affect the external validity of our results, because these participants over-represent food sources of a healthier dietary pattern. Thus, we can expect that the contribution from starchy foods to nutrient intake could be lower than that found in our sample and the contribution from sweets and pastry could be higher than that estimated by us.

Despite these limitations, our study is one of the few that has assessed the food sources of dietary intake in adolescents and the first in Portugal. To our knowledge, the articles that describe food sources in samples of adolescents are mostly from the 1990s and some of them describe food sources of only one nutrient ${ }^{(38-43)}$. Their results might be not comparable with our data, due to changes in food and nutrient intakes in recent decades $^{(44-46)}$ and changes in food sources of energy and nutrients ${ }^{(47,48)}$. In addition, our work is one of the few ${ }^{(49-51)}$ that, in this age group, explores food sources by social, demographic and behavioural characteristics of the participants. Two of those studies ${ }^{(49,51)}$ were published in 1986 and the other one ${ }^{(50)}$ only presents food sources according to participation in the National Food Lunch Program, in the USA. Since few data are published on this topic and because globalization appears to have contributed to the reduction in the differences in food intake between populations ${ }^{(52)}$, the data described here could be useful, not only for Portugal but also for health planning in other countries.

\section{Conclusions}

One quarter of energy intake was provided by starchy foods, and dairy foods plus meat accounted for another quarter. Starchy foods were also the major source of carbohydrates and fibre.

The contribution of meat to protein intake was more than double that of seafood, and meat was a major source of all types of fat. Sweets and pastry contributed more than $10 \%$ to the intakes of energy, carbohydrates, total and saturated fat.

Parental education was the characteristic most related with food sources and a higher education was associated with a higher contribution from healthier food groups.

\section{Acknowledgements}

This work was supported by grants from the Fundação para a Ciência e Tecnologia (POCTI/SAU-ESP/62399/ 2004). The authors declare no conflict of interests. J.A. drafted the first version of the manuscript, performed the statistical analysis and contributed to the interpretation of the analysis. M.S. performed the statistical analysis and contributed to the interpretation of the analysis. C.L. provided significant advice and contributed to the discussion of the manuscript. E.R. coordinated the manuscript and contributed to the interpretation and discussion of the analysis. All authors critically reviewed drafts of the manuscript and approved the final version of the manuscript.

\section{References}

1. World Health Organization (2008) 2008-2013 Action Plan for the Global Strategy for the Prevention and Control of Noncommunicable Diseases. Geneva: WHO; available at http://www.who.int/nmh/Actionplan-PC-NCD-2008.pdf

2. World Health Organization (2003) Diet, Nutrition and the Prevention of Chronic Diseases. Joint WHO/FAO Expert Consultation. WHO Technical Report Series no. 916. Geneva: WHO.

3. World Health Organization (2002) The World Health Report 2002. Reducing Risks, Promoting Healthy Life. Geneva: WHO; available at http://www.who.int/whr/2002/en/ whr02_en.pdf.

4. Thomas J (1991) Food choices and preferences of schoolchildren. Proc Nutr Soc 50, 49-57.

5. Shepherd R \& Dennison CM (1996) Influences on adolescent food choice. Proc Nutr Soc 55, 345-357.

6. World Health Organization \& Food and Agriculture Organization of the United Nations (1996) Preparation and Use of Food-based Dietary Guidelines. Report of a Joint FAO/ WHO Consultation. Geneva: WHO; available at http:// www.fao.org/docrep/X0243E/x0243e00.htm\#TopOfPage

7. Thompson FE, Sowers MF, Frongillo Jr EA et al. (1992) Sources of fiber and fat in diets of US women aged 19 to 50 : implications for nutrition education and policy. Am J Public Health 82, 695-702.

8. World Health Organization (2002) Food and Health in Europe: A New Basis for Action; Summary. Geneva: WHO; available at http://www.euro.who.int/document/e78578.pdf.

9. Prattala R, Hakala S, Roskam AJ et al. (2009) Association between educational level and vegetable use in nine European countries. Public Health Nutr 12, 2174-2182.

10. Vlismas K, Stavrinos V \& Panagiotakos DB (2009) Socio-economic status, dietary habits and health-related 
outcomes in various parts of the world: a review. Cent EurJ Public Health 17, 55-63.

11. Hulshof KF, Brussaard JH, Kruizinga AG et al. (2003) Socioeconomic status, dietary intake and 10 y trends: the Dutch National Food Consumption Survey. Eur J Clin Nutr 57, 128-137.

12. Thiele S, Mensink GB \& Beitz R (2004) Determinants of diet quality. Public Health Nutr 7, 29-37.

13. Ramos E \& Barros H (2007) Family and school determinants of overweight in 13-year-old Portuguese adolescents. Acta Paediatr 96, 281-286.

14. Willett WC, Sampson L, Stampfer MJ et al. (1985) Reproducibility and validity of a semiquantitative food frequency questionnaire. Am J Epidemiol 122, 51-65.

15. Amaral TF, Nogueira C, Paiva I et al. (1993) Weights and food portions. Rev Port Nutr 2, 13-23 (in Portuguese).

16. Lopes C, Fernandes PV \& Barros H (1994) Food frequency questionnaire - the effect of the food list extension on participants' classification. Arq Med 8, 291-294 (in Portuguese).

17. Lopes C (2000) Diet and myocardial infarction: a communitybased case-control study. PhD Thesis, University of Porto (in Portuguese).

18. Lopes C, Aro A, Azevedo A et al. (2007) Intake and adipose tissue composition of fatty acids and risk of myocardial infarction in a male Portuguese community sample. $J \mathrm{Am}$ Diet Assoc 107, 276-286.

19. Silva D, Rego C, Guerra A et al. (2004) Characterization of food habits and comparative study between two methods of food assessment in adolescents. Rev Aliment Humana 10, 33-40 (in Portuguese).

20. World Health Organization (1988) Measuring Obesity: Classification and Description of Anthropometric Data. Report on a WHO Consultation on the Epidemiology of Obesity. Copenhagen: WHO Regional Office for Europe.

21. Kuczmarski RJ, Ogden CL, Guo SS et al. (2002) 2000 CDC Growth Charts for the United States: methods and development. Vital Health Stat 11, 1-190.

22. Rodrigues SS \& de Almeida MD (2001) Portuguese household food availability in 1990 and 1995. Public Health Nutr 4, 1167-1171.

23. DAFNE - DAta Food NEtworking (2005) Trends within a country. http://www.nut.uoa.gr/DafnesoftWeb/Main.aspx? type $=$ trends (accessed March 2011).

24. He K, Song Y, Daviglus ML et al. (2004) Accumulated evidence on fish consumption and coronary heart disease mortality: a meta-analysis of cohort studies. Circulation 109, 2705-2711.

25. Trichopoulou A, Naska A \& Costacou T (2002) Disparities in food habits across Europe. Proc Nutr Soc 61, 553-558.

26. World Health Organization (1990) Diet, Nutrition and the Prevention of Chronic Diseases. WHO Technical Report Series no. 797. Geneva: WHO.

27. Lopes C, Oliveira A, Santos AC et al. (2006) Consumo alimentar no Porto. Porto: Faculdade de Medicina da Universidade do Porto; available at www.consumoalimentarporto.med.up.pt

28. Lien N, Lytle LA \& Klepp KI (2001) Stability in consumption of fruit, vegetables, and sugary foods in a cohort from age 14 to age 21. Prev Med 33, 217-226.

29. Smith KM \& Sahyoun NR (2005) Fish consumption: recommendations versus advisories, can they be reconciled? Nutr Rev 63, 39-46.

30. Marques-Vidal P, Ravasco P, Dias CM et al. (2006) Trends of food intake in Portugal, 1987-1999: results from the National Health Surveys. Eur J Clin Nutr 60, 1414-1422.

31. Moreira PA \& Padrao PD (2004) Educational and economic determinants of food intake in Portuguese adults: a crosssectional survey. BMC Public Health 4, 58.
32. Petkeviciene J, Klumbiene J, Prattala R et al. (2007) Educational variations in the consumption of foods containing fat in Finland and the Baltic countries. Public Health Nutr 10, 518-523.

33. Bandini LG, Schoeller DA, Cyr HN et al. (1990) Validity of reported energy intake in obese and nonobese adolescents. Am J Clin Nutr 52, 421-425.

34. Willett W (1998) Food-frequency methods. In Nutritional Epidemiology, 2nd ed., pp. 74-100. [W Willett, editor]. New York: Oxford University Press.

35. Hebert JR, Clemow L, Pbert L et al. (1995) Social desirability bias in dietary self-report may compromise the validity of dietary intake measures. Int J Epidemiol 24, 389-398.

36. Subar AF, Thompson FE, Smith AF et al. (1995) Improving food frequency questionnaires: a qualitative approach using cognitive interviewing. J Am Diet Assoc 95, 781-788.

37. Amanatidis S, Mackerras D \& Simpson JM (2001) Comparison of two frequency questionnaires for quantifying fruit and vegetable intake. Public Health Nutr 4, 233-239.

38. Albertson AM, Tobelmann RC \& Marquart L (1997) Estimated dietary calcium intake and food sources for adolescent females: 1980-92. J Adolesc Health 20, 20-26.

39. Basch CE, Shea S \& Zybert P (1992) Food sources, dietary behavior, and the saturated fat intake of Latino children. Am J Public Health 82, 810-815.

40. Moynihan P, Adamson A, Rugg-Gunn A et al. (1996) Dietary sources of calcium and the contribution of flour fortification to total calcium intake in the diets of Northumbrian adolescents. Br J Nutr 75, 495-505.

41. Nicklas TA, Farris RP, Myers L et al. (1995) Dietary fiber intake of children and young adults: the Bogalusa Heart Study. J Am Diet Assoc 95, 209-214.

42. Strain JJ, Robson PJ, Livingstone MB et al. (1994) Estimates of food and macronutrient intake in a random sample of Northern Ireland adolescents. Br J Nutr 72, 343-352.

43. O'Sullivan TA, Ambrosini G, Beilin LJ et al. (2011) Dietary intake and food sources of fatty acids in Australian adolescents. Nutrition 27, 153-159.

44. Alexy U, Sichert-Hellert W \& Kersting M (2002) Fifteen-year time trends in energy and macronutrient intake in German children and adolescents: results of the DONALD study. $\mathrm{Br}$ J Nutr 87, 595-604.

45. Nicklas TA, Demory-Luce D, Yang SJ et al. (2004) Children's food consumption patterns have changed over two decades (1973-1994): the Bogalusa Heart Study. J Am Diet Assoc 104, 1127-1140.

46. Stahl A, Vohmann C, Richter A et al. (2009) Changes in food and nutrient intake of 6- to 17-year-old Germans between the 1980s and 2006. Public Health Nutr 12, 1912-1923.

47. Cotton PA, Subar AF, Friday JE et al. (2004) Dietary sources of nutrients among US adults, 1994 to 1996. J Am Diet Assoc 104, 921-930.

48. Winkler G, Doring A \& Keil U (2000) Trends in dietary sources of nutrients among middle-aged men in southern Germany. Results of the MONICA Project Augsburg: dietary surveys 1984/1985 and 1994/1995. MONItoring trends and determinants in CArdiovascular disease. Appetite 34, 37-45.

49. Hackett AF, Rugg-Gunn AJ, Appleton DR et al. (1986) Dietary sources of energy, protein, fat and fibre in 375 English adolescents. Hum Nutr Appl Nutr 40, 176-184.

50. Gordon AR \& McKinney P (1995) Sources of nutrients in students' diets. Am J Clin Nutr 61, 1 Suppl., 232S-240S.

51. Kenney MA, McCoy JH, Kirby AL et al. (1986) Nutrients supplied by food groups in diets of teenaged girls. $\mathrm{J} \mathrm{Am}$ Diet Assoc 86, 1549-1555.

52. Iqbal R, Anand S, Ounpuu S et al. (2008) Dietary patterns and the risk of acute myocardial infarction in 52 countries: results of the INTERHEART study. Circulation 118, 1929-1937. 